\title{
Sensor-Based Recognition Fuzzy Mobile Robot Systems Designs
}

\author{
Shiang-Min Jou, Chih-Yung Chen ${ }^{1}$, Hsuan-Ming Feng,,", \\ Hua-Ching Chen ${ }^{2}$ and Ming-Hui Ho \\ Department of Electrical Engineering, Xiamen University, Xiamen 361005, P.R. China \\ ${ }^{1}$ Department of Computer and Communication, Shu-Te University, \\ Kaohsiung County 82445, Taiwan, R.O.C. \\ ${ }^{2}$ Department of Computer Science and Information Engineering, National Quemoy University, \\ No. 1 University Rd., Kin-Ning Vallage Kinmen, 892 Taiwan, R.O.C.
}

(Received July 2, 2014; accepted February 9, 2015)

Key words: particle swarm optimization, radio frequency identification, traveling salesman problems, fuzzy mobile robots, E-compass

Evolutionary particle swarm optimization (PSO) machines with hybrid sensors (i.e., radio frequency identification (RFID) and E-compass wireless network sensors) and the concepts of traveling salesman problems (TSPs) are applied to recognize the optimal routing paths in a dynamic space. Four active RFID tags and an E-compass indicate the robot position. The PSO algorithm with the guides of specific fitness functions simultaneously develops the proper fuzzy rules, avoids obstacles, and approaches the shortest routing path. The practice mobile robot control problems are considered to demonstrate the efficiency of the fuzzy control system. An illustrated simulation shows that the mobile robot system with the evolutional learning scheme can acquire the appropriate fuzzy rules to overcome multiple obstacles and achieve desired goals in dynamic and nonlinear environments.

\section{Introduction}

The fabrications of mobile robot platforms are actually integrated with embedded sensors, electrical power units, and motor gear driver modules. In addition, accurate software design and hardware implementation are efficiently generated by powerful computer technology and mechatronics engineering. ${ }^{(1)}$ Radio frequency identification (RFID) is known as a type of automatic wireless sensor identification system. Positive RFID tags with low power consumption, small size, and high portability are used to create a variety of transponder systems for solving various complex problems in robot applications. The perceptions of navigation systems with the associated aid of E-compass sensors are rapidly detecting the direction of a mobile robot in the hallway of

*Corresponding author: e-mail: hmfeng@nqu.edu.tw 
a building. The whole RFID and E-compass signatures easily obtain the control action of a mobile robot within a familiar space. ${ }^{(2-3)}$

Fuzzy logic ignores most of the mathematical computations needed to tackle such highly nonlinear and unexpected control problems. A fuzzy logic inference machine is implemented to simulate human thinking for solving robots' navigation task problems. Even the successful practical applications of mobile robots are developed on the basis of the fuzzy control theory; however, there are still some problems in selecting the perfect parameters for regulating mobile robot platforms. To eliminate the trial-and-error methods and improve the local optimal problems, particle swarm optimization (PSO) is used as an autonomous learning method. Defined parameters are proposed to accurately self-learn the mobile robot model for approaching the desired targets of the traveling paths in dynamic and vague environments. ${ }^{(4)}$ The near-optimal traveling path for mobile robot routing applications in unknown environments is similar to solving the traveling salesman problems (TSPs). The TSP solutions are perfectly utilized to decide the mobile robots' traveling sequence along the shortest tracking path. Therefore, the perfect traveling paths efficiently guide the mobile robot to follow the ordered points, and then approach toward the desired destination positions.

\section{Sensor-Based Robot Modeling and Controlled Structure}

The developed sensor-based mobile robot control system and its related modeling machines are illustrated in Fig. 1. Four modules include a motor driver, a mechanical machine, an E-compass, and a RFID reader, for constructing the main plant. A fuzzy system sends a motor driver module control signal to correctly regulate the moving

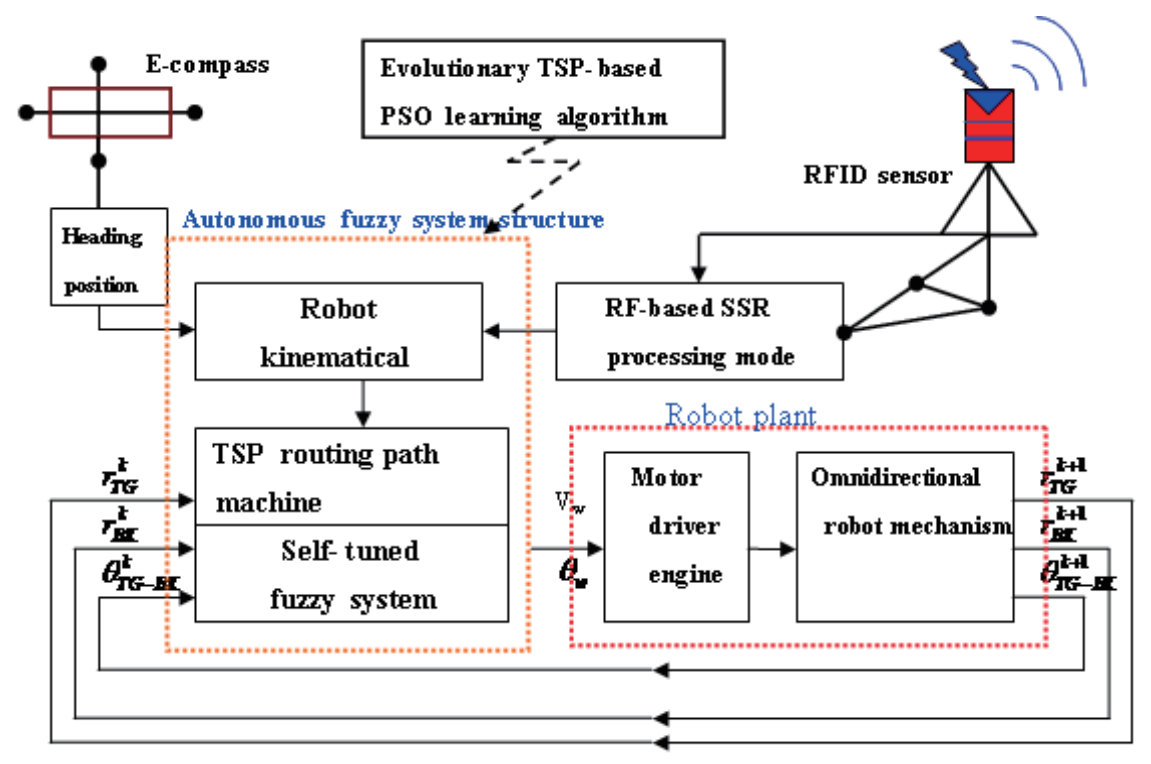

Fig. 1. (Color online) Hybrid evolutionary sensor-based recognition fuzzy mobile robot systems. 
speed and rotation angle of the omnidirectional gear scheme for approaching the desired targets. Both the RFID reader and E-compass are located at the front of the machine.

In the illustrated plot of Fig. 2, four active RFIDs stand on the rectangle region to calculate the current position of the robot.

$$
R S S=0.00018 r^{2}-0.20329 r+194.9196
$$

The robot easily obtains the distance $(r)$ from the estimated position $\left(x_{\mathrm{p}}, y_{\mathrm{p}}\right)$ of the coordinate system in a real situation. The estimated reader position of the mobile robot is achieved as the equivalent matrix form:

$$
\left[\begin{array}{l}
x_{\mathrm{p}} \\
y_{\mathrm{p}}
\end{array}\right]=\left(\boldsymbol{R}^{\mathrm{T}} \boldsymbol{R}\right)^{-1}\left(\boldsymbol{R}^{\mathrm{T}} \boldsymbol{S}\right)
$$

where $\boldsymbol{R}=\left[\begin{array}{ll}2\left(x_{\mathrm{B}}-x_{\mathrm{A}}\right) & 2\left(y_{\mathrm{B}}-y_{\mathrm{A}}\right) \\ 2\left(x_{\mathrm{C}}-x_{\mathrm{A}}\right) & 2\left(y_{\mathrm{C}}-y_{\mathrm{A}}\right) \\ 2\left(x_{\mathrm{D}}-x_{\mathrm{A}}\right) & 2\left(y_{\mathrm{D}}-y_{\mathrm{A}}\right)\end{array}\right], \quad \boldsymbol{S}=\left[\begin{array}{l}\left(r_{\mathrm{A}}\right)^{2}-\left(r_{\mathrm{B}}\right)^{2}+\left(x_{\mathrm{B}}^{2}+y_{\mathrm{B}}^{2}-x_{\mathrm{A}}^{2}-y_{\mathrm{A}}^{2}\right) \\ \left(r_{\mathrm{A}}\right)^{2}-\left(r_{\mathrm{C}}\right)^{2}+\left(x_{\mathrm{C}}^{2}+y_{\mathrm{C}}^{2}-x_{\mathrm{A}}^{2}-y_{\mathrm{A}}^{2}\right) \\ \left(r_{\mathrm{A}}\right)^{2}-\left(r_{\mathrm{D}}\right)^{2}+\left(x_{\mathrm{D}}^{2}+y_{\mathrm{D}}^{2}-x_{\mathrm{A}}^{2}-y_{\mathrm{A}}^{2}\right)\end{array}\right]$.

The mobile robot location is easily recognized using eq. (2). The E-compass is used to estimate the current direction in an unknown space. The target and obstacle positions are assigned in customized positions by a human through a personal digital assistant (PDA) machine. A hybrid RFID-based reader and E-compass sensors obtain the internal robot positions and then a fuzzy controller guides the robot machine to follow the prespecified path, and then the robot reaches the desired destinations by realizing the navigation information.

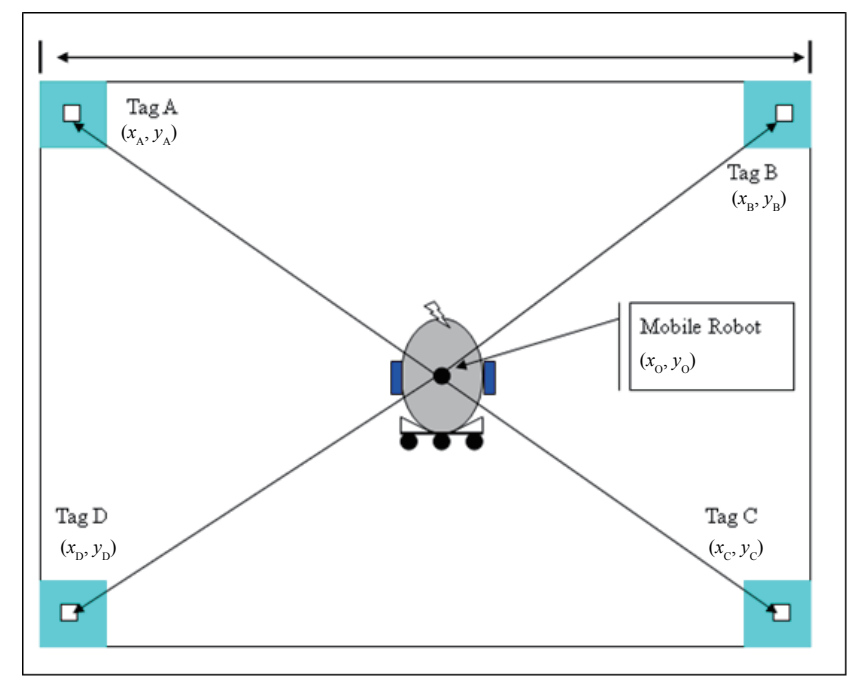

Fig. 2. (Color online) Four RFID reader tags in the navigation environment. 
In the configuration of the fuzzy mobile robot system, three input variables ( ${ }^{r} T G$, $\left.{ }^{r} B K,{ }^{\theta} T G-B K\right)$ and two output variables $\left(\theta_{\mathrm{w}}, v_{\mathrm{w}}\right)$ are selected and automatically developed using the PSO learning scheme to construct the fuzzy control structure. Here, $\theta_{\mathrm{w}}$ and $v_{\mathrm{w}}$ are denoted as the turn-rate angle of the motor wheel driver and the traveling line speed of the robot, respectively. The speed of the mobile robot is denoted as $v_{\mathrm{w}}^{k}$, and its turn-rate angle is set as $\theta_{\mathrm{w}}^{k}$ in the current time step $k$. The parameter selections of fuzzy system structures are selected using the TSP-based PSO learning algorithm, which is highly performed to simulate the sensor-based prototype of the mobile robot model in the next section.

\section{TSP-Routing-Based Fuzzy System Design through PSO Learning Algorithm}

The path-routing problem is utilized by the PSO for planning the sequences of the traveling stations. The TSP-based routing concept is considered as the same strategy in such mobile robot case to design the robot's shortest traveling sequences. If the possible index number is denoted as $[0.92,0.49,0.41,0.23,0.37,0.74,0.81]$ by the selection of the best particle's position at current time $(K)$, the related traveling sequence of the mobile robot is assigned as $(4 \rightarrow 5 \rightarrow 3 \rightarrow 2 \rightarrow 6 \rightarrow 7 \rightarrow 1)$. While the sequence table list is updated by the appropriate selection of the PSO learning algorithm in the next time step $(K+1)$, the new traveling path will be changed on the basis of the new order of the sequence list.(5) The routing-path solutions of the mobile robot can be considered to completely find better discrete combinations in constructing some possible sequences of the traveling path. Therefore, the various points' permutations in the choice of different combinations of integer sequences will be indicated as the traveling sequences. Various permutations with an adaptable creation of an integer sequence table will be assigned to transform the traveling path from the current time $(K)$ to the next time $(K+1)$. Owing to the direction of the well-defined fitness function, the evolutional PSO learning and path-routing scheme is applied to achieve the large table indexes with its self-evaluated learning scheme. If $Q$ denotes the total length in the traveling tour, the defined fitness function is used to evaluate the current selection as

$$
\text { Fitness }=\frac{k_{\mathrm{o}}}{\sum_{c i=1}^{N u m P-1}\left\|Q_{c i+1}\right\|-\left\|Q_{c i}\right\|} \text {. }
$$

Here, NumP denotes the total number of traveling points, $k_{\mathrm{O}}$ is a positive constant assigned by the designer, and $\|\cdot\|$ indicates the length symbol. It is clear that the higher fitness value presents the shorter distance of the selected traveling path with the sequence list of points to approach the target.

In the design of the TSP mobile robot fuzzy system structure, the whole input variables are determined by an $n$-dimensional data vector in the form of $\boldsymbol{X}=\left(x_{1}, x_{2}, \ldots\right.$, $\left.x_{n}\right)$. A single tone but two variables of $Y$ are generated to obtain the control output. The fuzzy rules are described as the formulas: ${ }^{(6)}$ 


$$
R U L E^{(i)}: \text { IF } \boldsymbol{X} \text { is } H E_{i} \text { THEN } U \text { is } u_{m}^{i}, i=1,2, \ldots, M U N ; m \in\{1,2\},
$$

where $M U N$ is the total number of fuzzy rules and $u_{m}^{i}$ denotes a real value of the corresponding $i$-th rule. Those membership functions $\left(H E_{i}\right)$ are determined by

$$
H E_{l}(\boldsymbol{X})=\exp \left\{-\left[\sum_{l=1}^{n} \frac{\left(x_{l}-c_{l}^{i}\right)^{2}}{\left(\delta_{l}^{i}\right)^{2}}\right]\right\}
$$

where $c_{l}^{i}$ and $\delta_{l}^{i}$ are considered as the center and length of the $l$-th principal axis for the $i$-th hyper-ellipsoid membership function, respectively. Both required parameters $c_{l}^{i}$ and $\delta_{l}^{i}$ are regulated to approach appropriate fuzzy rules in the premised part, and $u_{m}^{i}$ is the consequent parameter of the fuzzy system. The simple singleton fuzzy inference and the weighted average defuzzifier method are proposed in this study. That is, while the input vector $\boldsymbol{X}$ is applied to the fuzzy system, the output value $\left(Y_{0}\right)$ is derived as

$$
Y_{0}=\frac{\sum_{i=l}^{M} H E_{i}(\boldsymbol{X}) \cdot u_{m}^{i}}{\sum_{i=l}^{M} H E_{i}(\boldsymbol{X})},
$$

where $M$ is the active fuzzy number. Since the selected contour of the $i$-th membership function $H E_{i}(\boldsymbol{X})$ is derived by the combination of parameters $\left\{c_{1}^{i}, \delta_{1}^{i}, c_{2}^{i}, \delta_{2}^{i}, \ldots, c_{n}^{i}, \delta_{n}^{i}\right\}$ and the real value $u_{m}^{i}$, the available parameter selection affects the fuzzy mobile robot system to a large extent.

An appropriate combination of parameter sets is selected using the evolutionary PSO algorithm. The MAX-LENGTH selection process will lead to a large selection to achieve the same vector length in all the particles. ${ }^{(7)}$ The FIT_ROB( $\left.\boldsymbol{R}_{i}\right)$ is the evaluated fitness value, which can be determined to select the best input parameter $\boldsymbol{R}_{i}$.

$$
F I T_{-} R O B\left(\boldsymbol{R}_{i}\right)=\left(\frac{25}{R M S E}+\frac{7}{M}\right) * R T S
$$

Here, RMSE is the root-mean-square error of the distance between the destination and initial positions. The RTS indicates the robot tracking state, which is set as 1 , while the mobile robot does not collide with a block. Otherwise, the other tracking state of RTS is 0 when the mobile robot unsuccessfully goes through the obstacles in the possible traveling path. The positive number $(M)$ is denoted as the actual fuzzy number. The goals of the PSO learning algorithm can be determined by obtaining the maximal fitness value. The procedure of the TSP-based PSO learning algorithm is described by the following steps:

TPSO1: Set the maximal iteration number and initialize it as 0 . Set the learning rate 
$\left(\alpha_{1}, \alpha_{2}\right)$. Randomly generate the initial particle's parameters $\left(\boldsymbol{R}_{j}\right)$ and the fuzzy number list $\gamma_{j}$.

TPSO2: Create the initial structure of the fuzzy control system from the $\boldsymbol{R}_{j}^{*}$. The active membership function of each individual particle is proposed with respect to the $j$-th $l_{j n}$. It is described by $\boldsymbol{R}_{j}^{*}=\left[l_{j n} \boldsymbol{R}_{j}\right]$. Here, $l_{j n}$ is randomly generated by

$$
l_{j n}=R O D\left\{L_{j n}+\left(U_{j n}-L_{j n}\right) * \operatorname{rand}()\right\} .
$$

$R O D$ is the round operator that generates $l_{j n} \in\left\{L_{j n}, L_{j n}+1, \ldots, U_{j n}\right\}$ and $\operatorname{rand}()$ denotes the random number, which is uniformly distributed in the interval $[0,1]$.

TPSO3: Find the active selection based on the comparison of the list $l_{j n}$.

TPSO4: Run the TSP process to control the fuzzy mobile robot in an individual area.

TPSO5: Execute the PSO learning algorithm to regulate the parameters of the fuzzy system.

TPSO6: Determine the individual fitness value of each particle using eq. (7).

TPSO7: Select the new $P_{\text {best }}^{\text {new }}$, which obtains the largest fitness value with the comparison of each individual's fitness value and the previous personal best $P_{\text {best }}^{\text {old }}$.

TPSO8: Renew the solution of the global best particle $\left(G_{\text {best }}^{\text {new }}\right)$, which contains the highest fitness value from the amount of $P_{\text {best }}^{\text {new }}$.

TPSO9: $g=g+1$.

TPSO10: If $g$ equals the terminal iteration, then go to exit; otherwise, go to step TPSO4.

TPSO11: The best particle's parameters $\boldsymbol{R}_{j}^{\text {best }}$ will be selected to automatically develop the best mobile robot fuzzy control system.

\section{Implementations and Conclusions}

In the design of the hardware mobile robot, two photos in front and vertical views of practice robot architectures are actually presented in Fig. 3. Three omnidirectional wheels are set under a big circle plastic plate. The operator handles the PDA machine to plan the RFID sensor positions and TSP-based routing path, where four yellow rectangular areas are denoted as the active RFID sensors for self-localization. Three red circles are the block areas that are proposed to escape. Three green rectangle points are mentioned as the traveling and going traces using the designed TSP-based travel routing scheme. The compass provides an estimate of the robot rotational motion in a short response time. The fuzzy control system with the evolutional PSO learning method is proposed to estimate the mathematical model of the mobile robot machine. Approximate fuzzy rules are determined to make a suitable control signal, which regulates the motor gear drive into the desired point.

The four RFID stations are located at the corner of the rectangle moving space; its width and length are 280 and $320 \mathrm{~cm}$, respectively. To demonstrate the adaptability of the actual mobile robot machine, three obstacles and three intermediate target positions are actually defined in advance. The robot's dynamic trace with 16 sequence images is shown in Fig. 4. Here, the green point is a starting position, three red-white points are 


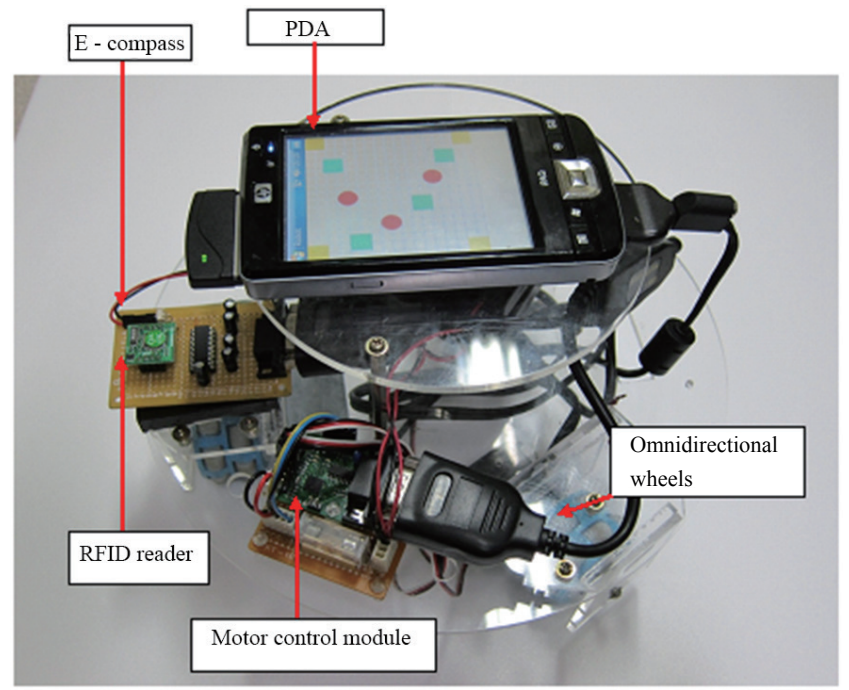

Fig. 3. (Color online) Vertical view of the practice mobile robot structure.
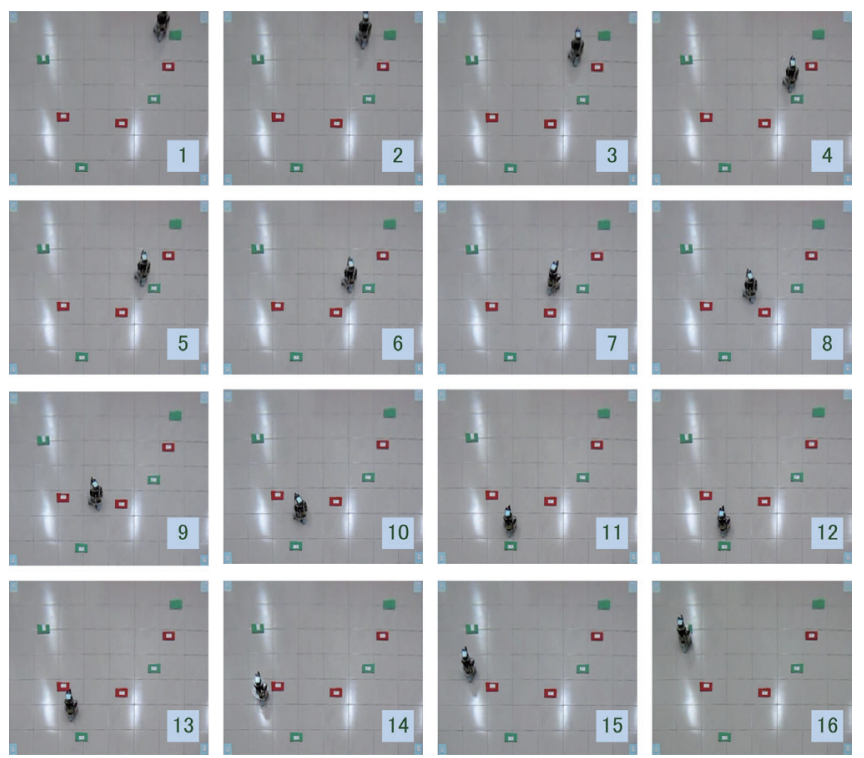

Fig. 4. (Color online) Mobile robot moving scenes with 16 sequence images.

obstacles, and green-white points are intermediate destinations. The practice mobile robot with the PSO-trained fuzzy control rules can actually guide the mobile robot platform, approximating the intermediate destinations, escaping the obstacles, and stopping in the desired end position. 


\section{Acknowledgements}

This research was partly supported by the National Science Council of the Republic of China under contract Nos. NSC 99-2221-E-507-004 and 100-2221-E-507-002.

\section{References}

1 C. T. Chi, Y. T. Wang, S. T. Cheng and C. A. Shen: Sens. Mater. 26 (2014) 353.

2 A. Sipahioglua, A. Yazici, O. Parlaktuna and U. Gurel: Rob. Auton. Syst. 56 (2008) 289.

3 Y. L. Chen and X. Z. Shi: Int. J. Eng. Technol. Innovation 2 (2012) 216.

4 R. Tesoriero, R. Tebar, J. A. Gallud, M. D. Lozano and V. M. R. Penichet: Expert Syst. Appl. 37 (2010) 894.

5 H. M. Feng and K. L. Liao: Inf. Sci. 270 (2014) 204.

6 H. M. Feng, C. Y. Chen and J. H. Horng: Expert Syst. Appl. 37 (2010) 4009.

7 H. M. Feng: Neurocomputing 70 (2006) 241. 